\title{
Simultaneous bilateral implantation of ex-press glaucoma shunt for secondary glaucoma treatment due to axenfeld-rieger syndrome: a case report
}

\begin{abstract}
Background: Axenfeld-Rieger Syndrome is a rare genetic disease affecting multiple organ systems. In the eye, it can be manifested with varying degrees of anterior segment dysgenesis and it carries a high risk of glaucoma.

Methods: We describe a case of 23 year old male patient with Axenfeld-Rieger syndrome who had bilateral cataract surgeries with IOL/iris diaphragm implantation prior to the referral. After those procedures he developed high and uncontrolled intraocular pressure (IOP) on both eyes. Since the patient was from the rural part of the country, we decided to perform same day bilateral glaucoma surgery with Ex-Press glaucoma shunt implantation. Both surgeries were done by the same surgeon.

Results: At first postoperative day, both eyes had slight hypotony, so the additional viscoelastic has been instilled in the anterior chambers to maintain the pressure. There was no retinal, nor choroidal detachment. Throughout the follow up period, the IOP was stabile without any additional medications, visual acuity improved significantly and there were no other postoperative complications

Conclusion: Ex-Press glaucoma shunt can be very useful in treating secondary glaucoma due to rarely seen genetic diseases which can affect eyes, and it can be safely implanted as a same day bilateral procedure.
\end{abstract}

Keywords: axenfeld-rieger, intraocular pressure, glaucoma, shunt
Volume 3 Issue 2 - 2015

\author{
Bojan Kozomara,' Ratimir Lazic, ${ }^{2}$ Ernesta \\ Potkonjak,' Maja Bohac,' Nikica x Nikica \\ Gabric $^{2}$ \\ 'Eye Hospital "Svjetlost” Banja Luka, Bosnia-Herzegovina \\ 'University Eye Hospital "Svjetlost”, Croatia
}

Correspondence: Bojan Kozomara, Eye Hospital "Svjetlost" Banja Luka, Bul. Desanke Maksimovic I2, 78000 Banja Luka, RS Bosnia-Herzegovina, Fax +387 51439778 ,

Email bojan@svjetlost-banjaluka.ba

Received: October 13, 2015 | Published: November 04, 2015
Abbreviations: IOP, intraocular pressure; ARS, axenfeldrieger syndrome; FOX, forkhead box; IOL, chamber intraocular lens; BSCVA, best spectacle corrected visual acuity

\section{Introduction}

Axenfeld-Rieger Syndrome (ARS) is a rare genetic disease affecting multiple organ systems. In the eye, it can be manifested with varying degrees of anterior segment dysgenesis and it carries a high risk of glaucoma. ${ }^{1}$ Recent advantages in molecular genetics have identified two major genes, PITX2 and FOXC1, demonstrating a wide spectrum of mutations, which aids in the molecular diagnosis of the disease, although evidence exists to implicate other loci in this condition. ${ }^{2}$ The management of individuals affected by AxenfeldRieger syndrome requires a multidisciplinary approach and would include dedicated surveillance and management of glaucoma, sensorineural hearing loss, and cardiac, endocrinological, craniofacial and orthopaedic abnormalities. This syndrome was first described by Axenfeld in 1920's in a patient whose ocular abnormality was a white line in the posterior aspect of the cornea, near the limbus, with tissue strands extending from peripheral iris to this prominent line. Beginning in the mid-1930's, Rieger reported cases with similar anterior segment anomalies, but with additional changes in the iris, including corectopia, atrophy, and hole formation. It was also discovered that some of these patients had associated non ocular development defects, especially of the teeth and facial bones. Axenfeld referred to his case as posterior embryotoxon of the cornea, while Rieger used the term mesodermal dysgenesis of the cornea and iris. ${ }^{3}$

Patients with Axenfeld-Rieger syndrome typically have juvenile-onset or early adult onset (before the age of 40) glaucoma. Histopathological examination of ARS eyes revealed malformations in the trabecular meshwork and Schlemm's canal, both of which are essential $f$

or the proper drainage of aqueous humor from the anterior chamber of the eye to occur. Inefficient drainage of the aqueous humor often results in elevated intraocular pressure (IOP), which is a major risk factor for the development of glaucoma.

Mutations in the Forkhead Box C1 (FOXC1) gene are associated with ARS and glaucoma. FOXC1 belongs to the Forkhead box (FOX) family of transcription factors, which share and evolutionary conserved DNA-binding domain known as the Forkhead domain. ${ }^{4}$ The vast majority of FOXC1 missense mutations identified in ARS patients are located within the fork-head domain, highlighting the importance of an intact DNA-binding domain for FOXC1 to function during ocular development. In addition to FOXC1's role during ocular development, FOXC1 appears to have a protective role in the adult eye. FOXC1 is expressed in the adult eye including the trabecular meshwork. More specifically, FOXC1 maintains homeostasis in the adult trabecular meshwork by regulating anti-apoptotic genes such as FOXO1a. $^{5}$ 
Even though there is still no specific data on the prevalence of glaucoma in ARS, as well as the anomaly itself, some there are certain variations found in the literature ranging from $8 \%$ to $15 \%{ }^{6}$ This article shows a case of 23 year old male patient with Axenfeld-Rieger syndrome, to whom the same day bilateral shunt implantations were performed without any postoperative complications and with good outcome.

\section{Methods}

A 23 year old male patient was referred to our clinic from another rural eye center due to uncontrolled intraocular pressure (IOP). Values of the IOP were $36 / 37 \mathrm{~mm} \mathrm{Hg}$ on triple topical therapy, which included beta blockers, Carboanhydrase inhibitors and latanoprosts. One year prior to the referral, the patient had been diagnosed with AxenfledRieger syndrome and underwent bilateral phacoemulsification surgery. Because of the iris atrophy and atopic pupils, during the same procedure he had been implanted with posterior chamber intraocular lens (IOL) with iris diaphragm. Three months postoperatively, YAG laser capsulotomies were also performed on both eyes.

On the preoperative examination, best spectacle corrected visual acuity (BSCVA) on the right eye was 0.2 (Snellen) with $-5.50 \mathrm{x}-2.25$ /170, while the left eye had the BSCVA of 0.4 with $-4.75 \mathrm{x}-1.50 / 165$. Slit lamp examination showed significant chronic conjunctival hyperemia, clear cornea and no inflammatory cells in the anterior chamber. Intraocular lens and iris diaphragm were well positioned. YAG laser capsulotomies of no more than $3 \mathrm{~mm}$ in diameter were also clearly visible. Fundus examination reviled significant optic nerve cut to disk ratio $(\mathrm{C} / \mathrm{D})$, which was confirmed by the optic nerve OCT scan, where the $\mathrm{C} / \mathrm{D}$ for the right eye was 0.855 , while the left eye's $\mathrm{C} / \mathrm{D}$ was 0.725 . There was no macular edema due to prolonged latanoprost therapy.

Since the patient lived in rural area and is not able to attend regular eye examinations, we have decided to undergo same day bilateral glaucoma surgery with Ex-Press glaucoma shunt implantation in both eyes. All surgeries have been performed by the same surgeon (RL). After dissection of the conjunctiva and scleral flap creation, anterior chamber was penetrated with $23 \mathrm{G}$ needle, after which the shunt was positioned and secured. Prior to the flap repositioning, topical $0.02 \%$ mitomycin $\mathrm{C}$ was applied for one minute. At the end, the flap was closed with three $9 / 0$ nylon interrupted sutures, while conjunctiva was closed with one $8 / 0$ vicryl continuous suture. Function of the shunt was tested with injection of methylcellulose viscoelastic through temporal corneal paracenthesis. Intentionally, both eyes were left slightly hypertonic, because we tried to avoid sudden hypotony and retinal and/or choroidal detachment. Postoperatively, patient received combination of antibiotic and corticosteroid eye drops for one week, while postoperative examinations were every day for the first week, and then every month for the next six months.

\section{Results}

At day one, even though we have inflated both eyes intraoperatively with viscoelastic, there was significant hypotony of $5 \mathrm{mmHg}$ for the right eye and $6 \mathrm{mmHg}$ for the left eye. However, there was no bleb, or anterior chamber inflammations, while cornea had a few Descemet folds with a discrete stromal edema. We have decided to instill more additional viscoelastic into the anterior chamber in both eyes, in order to prevent possible retinal/choroidal detachments. This action proved correct, and the next day the IOP normalized with the values of $19 / 20 \mathrm{mmHg}$ respectfully. For the next six days the IOP was stable, with the value of $14 / 15 \mathrm{mmHg}$ on day two to $15 / 13 \mathrm{mmHg}$ on day seven. The bleb and the filtration bed were functioning properly throughout the first week of follow up. On day seven, patient's visual acuity was measured, which improved significantly comparing to the preoperative values. The BSCVA was 0.5 (Snellen) on the right eye and 0.7 Snellen on the left eye, with the same preoperative refraction.

Visual acuity remained stable throughout the follow up period of six months, together with the values of intraocular pressure. All topical anti glaucoma therapy was discontinued. Overall patient satisfaction was outstanding, mainly because the visual acuity improved drastically. The only complaint was opacities in the vitreous, particularly on the right eye.

\section{Discussion}

Even though Axenfeld-Rieger syndrome (ARS) is a rare and yet not well understood hereditary disease, some improvements in the mechanism detection have been achieved recently. ${ }^{7}$ Human genetics now offers some more answers on gene mutations associated with this syndrome, where some authors in their recent studies state that the PITX2 gene plays an important role in activation and development of symptoms in AR syndrome. Others emphasize that mutations on CYP1B1, subtype of cytochrome P450 are responsible for glaucoma development, including patients with ARS. ${ }^{8}$ Treatment options in these cases are still narrowed to either topical or filtration surgeries. As it is well known, there are still two basic options when it comes to the surgery, which include classic trabeculectomy with or without topical $0.02 \%$ mitomycin $\mathrm{C}$ application, or filtration surgery with glaucoma shunt implantation. In our case, we have decided to use the second option because the patient had previous cataract surgery with artificial iris implantation and was on the triple topical therapy. This means that classic trabeculectomy could not be performed due to lack of natural iris and without certainty that the results would be satisfactory. However, there are other implant options today that can also have good postoperative results in the IOP control. ${ }^{9}$ Nevertheless, some surgeons still prefer good old trabeculectomy as a treatment of choice, with rather excellent postoperative results. ${ }^{10}$

Better postoperative patient's visual acuity results were expected, but not so significantly. With the same refractive error, the BSCVA improved rapidly, and increased from 0.2 to 0.5 (Snellen) on the right eye, and from 0.4 to 0.7 (Snellen) on the left eye. Logical explanation for this improvement can be found in two very specific circumstances; first one being age of the patient and the ability of the optic nerve to sustain good blood flow with elevated IOP for a longer time period than in elderly patients; the second being improvement in and consequent regression of stromal edema caused by preoperative uncontrolled intraocular pressure and endothelial pump dysfunction. Radhakrishnan et al. ${ }^{9}$ in their case report state maintenance of the same BCVA of 20/30 one year post-op, while Parikh et al. ${ }^{10}$ advocate stabile IOP in their patient without any additional topical therapy for more than 6 months.

\section{Conclusion}

Glaucoma shunt can be safely used as a therapeutic option for better control of intraocular pressure in embryotoxon and AxenfeldRieger syndrome. Surgery can be performed bilaterally as a same day surgery without any risks to serious postoperative complications. Future studies on large samples are needed to support these findings. 


\section{Acknowledgments}

None.

\section{Conflicts of interest}

The author declares there is no conflict of interest.

\section{References}

1. Yang HJ, Lee YK, Joo CK, et al. A family with Axenfeld-Rieger syndrome: report of the clinical and genetic findings. Korean $J$ Ophthalmol. 2015;29(4):249-255.

2. Chang TC, Summers CG, Schimmenti LA, et al. Axenfeld-Rieger syndrome: New perspectives. Br J Ophthalmol. 2012;96(3):318-322.

3. Shields MB. Axenfeld-Rieger syndrome: a theory of mechanism and distinctions from the iridocorneal endothelial syndrome. Trans Am Ophthalmol Soc. 1983;81:736-784.

4. Kim GN, Ki CS, Seo SW, et al. A novel forkhead box C1 gene mutation in a Korean family with Axenfeld-Rieger syndrome. Mol Vis. 2013;19:935943.
5. Strungaru MH, Dinu I, Walter MA. Genotype-phenotype correlation in Axenfeld-Rieger malformation and glaucoma patients with FOXC1 and PITX2 mutations. Invest Ophthamol Vis Sci. 2007;48(1):228-237.

6. Shields MB. Axenfeld-Rieger syndrome: a theory of mechanism and distinctions from the iridocorneal endothelial syndrome. Trans Am Ophthalmol Soc. 1983;81:736-784.

7. Ito YA, Walter MA. Genomics and anterior segment dysgenesis: a review Clin Experiment Ophthalmol. 2014;42(1):13-24.

8. Pasutto F, Mauri L, Popp B, et al. Whole exome sequencing reveals a novel de novo FOXC1 mutation in a patient with unrecognized AxenfeldRieger syndrome and glaucoma. Gene. 2015;568(1):76-80.

9. Radhakrishnan OK, Pahuja K, Patel K, et al. OLOGEN ${ }^{\circledR}$ implant in the management of glaucoma in an unusual case of Axenfeld-Rieger syndrome. Oman J Ophthalmol. 2014;7(2):90-92.

10. Parikh RS, Parikh SR, Debashish B, et al. Unusual presentation of Axenfeld-Rieger syndrome. Indian J Ophthalmol. 2011;59(4):312-314. 